\title{
Radiation-associated angiosarcoma after autologous breast reconstruction: report of two cases in a plastic surgery unit
}

\author{
Christina Yip ${ }^{1,2} \cdot$ Eva Weiler-Mithoff $^{1} \cdot$ Julie Claire Doughty ${ }^{2} \cdot$ Steven J. Lo ${ }^{1}$
}

Received: 5 February 2019 / Accepted: 30 April 2019 / Published online: 30 May 2019

(C) The Author(s) 2019

\begin{abstract}
Radiation-associated breast angiosarcoma is a rare diagnosis but is commonly reported. Angiosarcoma occurring in nonbreast tissue in breasts that have been reconstructed with autologous tissue following mastectomy is extremely rare. In our unit, we have managed two patients with angiosarcoma arising in non-breast tissue autologous reconstructions. Our report emphasises that any tissue treated with radiotherapy - that is, the residual mastectomy skin flaps or non-breast tissue autologous reconstructions - are at a risk of secondary angiosarcoma. We also discuss recommended surgical management, surveillance and genetic testing.

Level of Evidence is V, risk study.
\end{abstract}

Keywords Radiation $\cdot$ Angiosarcoma $\cdot$ Breast reconstruction

Radiation-associated breast angiosarcoma is a rare diagnosis but is commonly reported. Angiosarcoma occurring in non-breast tissue in breasts that have been reconstructed with autologous tissue following mastectomy is extremely rare. Thus far only one case has been reported in the literature [1]. In our unit, we have managed two patients with angiosarcoma arising in nonbreast tissue autologous reconstructions. Our report emphasises that angiosarcoma can arise in breasts that have been treated with mastectomy - in residual skin flaps and in the tissue from the reconstructions themselves. We also discuss recommended surgical management, surveillance and genetic testing.

Christina Yip

christinaoyyip@gmail.com

1 Canniesburn Plastic Surgery Unit, Glasgow Royal Infirmary, 84 Castle Street, Glasgow G4 OSF, UK

2 Department of Breast Surgery, Gartnavel General Hospital, 1053 Great Western Road, Glasgow G12 0XP, UK

\section{Case 1}

A 61-year-old patient was diagnosed with bilateral screendetected breast cancer and required bilateral mastectomy, axillary node sampling and immediate latissimus dorsi reconstruction in 2003. Her pathology report suggested a more advanced tumour on the right (grade 1, oestrogen positive, invasive ductal carcinoma of $22 \mathrm{~mm}$ ), with one out of seven right axillary lymph nodes involved; she therefore required adjuvant radiotherapy (50 Gy in 25 fractions) to her right reconstructed breast and right supraclavicular fossa.

Five years post-radiotherapy, she developed a 'bruise' to the lower inner quadrant of her right reconstructed breast, over the mastectomy skin flap (Fig. 1). In addition to the discoloured area, there was also a small skin nodule which was clinically suspicious of angiosarcoma. An excision biopsy confirmed the diagnosis. A neo-mastectomy was warranted and the patient had chest wall reconstruction using a transposition flap (Fig. 2). This patient was a poor candidate for free abdominal tissue transfer since she had multiple abdominal surgeries for ulcerative colitis and was left with significant rectus diastasis and extensive scarring to her lower abdomen. She also suffered from asthma and did not favour a microsurgical reconstruction. Her neo- 


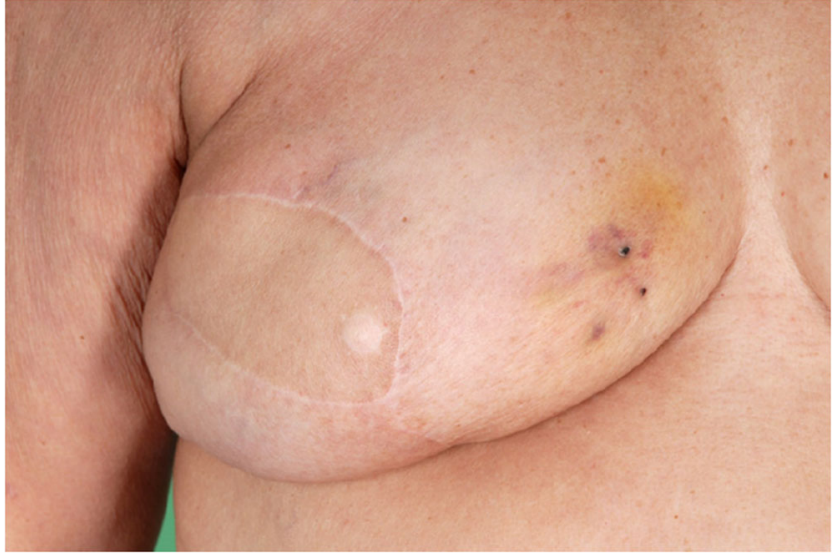

Fig. 1 Five years post-radiotherapy, patient developed skin discolouration and a small necrotic skin nodule to the lower inner quadrant of her right reconstructed breast, over the mastectomy skin flap

mastectomy was curative for angiosarcoma and she required no further treatment. A year after her chest wall reconstruction, the patient opted for follow-up locally by the breast surgery team. To date the patient remains disease free.

\section{Case 2}

A 71-year-old patient was diagnosed with multifocal, grade 2, oestrogen and progesterone positive, invasive ductal carcinoma of her left breast in 2007. She underwent left mastectomy, axillary node clearance ( $3 / 13$ positive nodes) and immediate deep inferior epigastric perforator (DIEP) flap reconstruction. Post-surgery, she required adjuvant chemotherapy, two field radiotherapy (50 Gy in 25 fractions) to her left reconstruction and hormonal (anastrozole) therapy. In 2011, she developed a 'bruise' to the lower inner quadrant of her left reconstructed breast. A skin biopsy was taken and suggested benign papillary haemangioma only. The discolouration settled after the biopsy, and 5 years later (April 2017), she re-presented with a rash to the lower inner quadrant of her left reconstructed breast (Fig. 3a). A core biopsy and an excision skin biopsy were performed, which suggested a vascular process, not typical of angiosarcoma. After the biopsies, the rash had almost completely gone and the patient was then kept under close observation once every 2 months. By November 2017, she returned with a widespread purple discolouration to the skin paddle of her DIEP reconstruction that extended onto the lower mastectomy skin flap (Fig. 3b). Repeat excision biopsies suggested the presence of malignant spindle cell proliferation, which stained positively for CD31 and CD34, confirming the diagnosis of angiosarcoma. This patient was referred to the

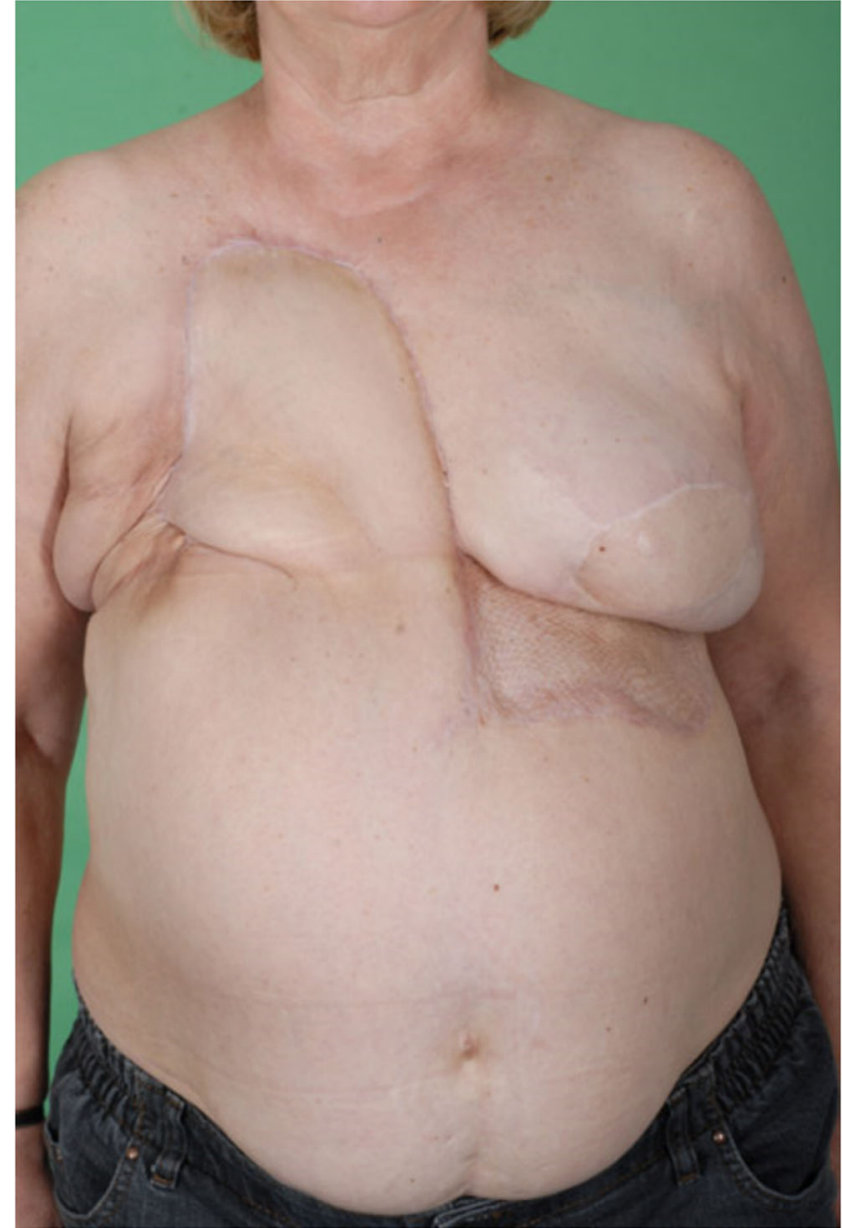

Fig. 2 A transposition flap was required to reconstruct right anterior chest wall defect, following neo-mastectomy

sarcoma multidisciplinary team and she required neo-mastectomy, frozen sections and immediate chest wall reconstruction with free anterolateral thigh (ALT) flap (Fig. 4). Pathology confirmed complete excision of high-grade angiosarcoma; no further treatment was indicated. Since surgery, she has been kept under regular surveillance by the sarcoma team.

\section{Discussion}

Secondary angiosarcoma of the breast, as a result of previous radiotherapy following breast conservation surgery or mastectomy, is very rare, with a reported incidence of $0.1-0.2 \%$ per year [2]. Although radiation to the breast was used therapeutically, it can also lead to DNA damage, resulting in genome instability, which in selected cases 

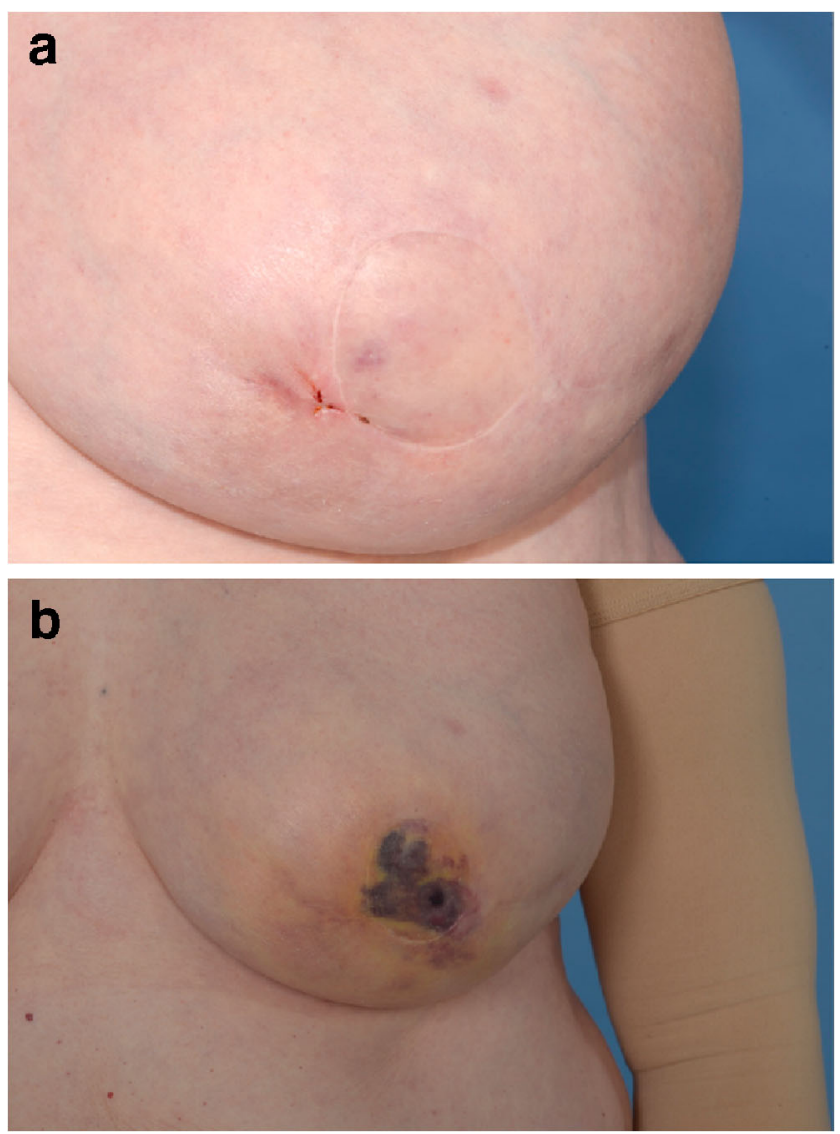

Fig. 3 a Ten years post-radiotherapy, patient re-presented with a rash to the lower inner quadrant of her left reconstructed breast, over the mastectomy skin flap. b Six months following initial biopsy, patient developed a widespread purple discolouration to the skin paddle of her DIEP reconstruction that extended onto the lower mastectomy skin flap

may lead to the formation of sarcoma [2]. It may have a latency period of several years prior to presentation and the initial clinical signs can be very subtle, as demonstrated in our cases. Radiotherapy contributes to a 'field effect' in all previously irradiated tissue; hence, clinical signs of angiosarcoma can appear on the mastectomy skin flap or on the skin paddle of the abdominal flap. It is vital to have a high index of suspicion, close monitoring of the changes in clinical signs and repeat biopsies as necessary, in order to diagnose secondary angiosarcoma.

Feinberg et al. recently reviewed the impact of early specialist management on survival from angiosarcoma and suggested that patients managed initially by the sarcoma team have fewer local recurrences and significant improved disease-specific survival [3]. Higher rates of positive margins

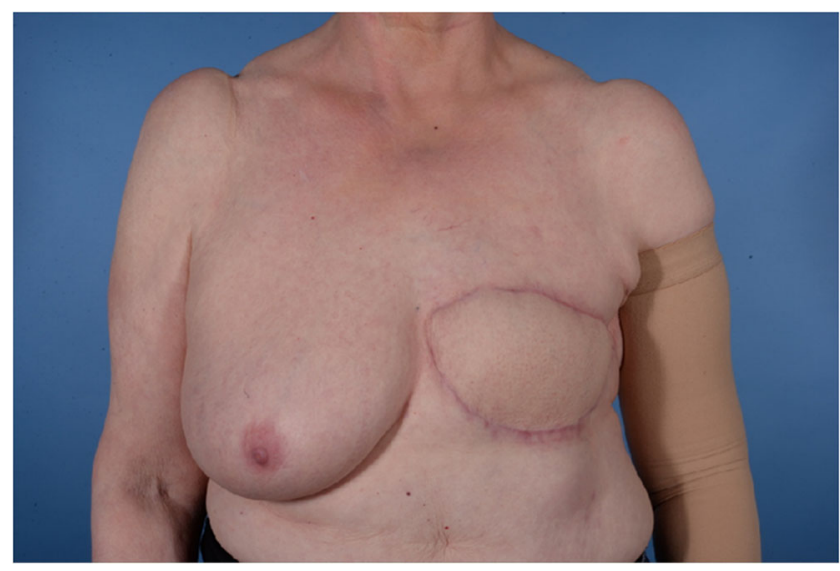

Fig. 4 A free anterolateral thigh (ALT) flap was used to reconstruct left anterior chest wall defect, following neo-mastectomy

have been seen with wide local excision, and specialist sarcoma units such as the Royal Marsden suggest radical excision [4]. Excision of the field change associated with previously irradiated tissue may lead to better local control, reducing the incidence of future local recurrence [3]. Our surgical approach to radiation-associated angiosarcoma is similar to Feinberg et al.: we advocate radical neo-mastectomy rather than wide local excision, frozen section for margin analysis and chest wall reconstruction. Follow-up is in keeping with the Scottish Sarcoma National Follow-Up guidelines with 4 monthly clinical assessment, chest X-ray and annual regional imaging [5]. Genetic testing should be considered in this group of patients, as pre-existing genetic abnormalities such as BRCA and p53 mutations may potentiate the carcinogenic effects of irradiation, leading to secondary angiosarcoma [6-8].

The cases we describe here indicate that angiosarcoma can occur in breasts that have been treated with mastectomy, and that breast tissue itself is not a pre-requisite for angiosarcoma formation. Any tissue treated with radiotherapy - that is, the residual mastectomy skin flaps or non-breast tissue autologous reconstructions-are at a risk of secondary angiosarcoma. It is essential that the treating breast clinician is aware of this rare but critical possibility.

\section{Compliance with ethical standards}

Conflict of interest None.

Ethical approval Not applicable.

Informed consent Obtained and consented. 
Open Access This article is distributed under the terms of the Creative Commons Attribution 4.0 International License (http:// creativecommons.org/licenses/by/4.0/), which permits unrestricted use, distribution, and reproduction in any medium, provided you give appropriate credit to the original author(s) and the source, provide a link to the Creative Commons license, and indicate if changes were made.

\section{References}

1. Hanasono M, Osborne M, Dielubanza EJ, Peters SB, Gayle LB (2005) Radiation-induced angiosarcoma after mastectomy and TRAM flap breast reconstruction. Ann Plast Surg 54(2):211-214

2. Duncan MA, Lautner MA (2018) Sarcomas of the breast. Surg Clin North Am 98(4):869-876

3. Feinberg L, Srinivasan A, Singh JK, Parry M, Stevenson J, Jeys L, Grimer R, Peart F, Warner R, Ford S, Gourevitch D, Hallissey M, Desai A (2018) Impact of specialist management on survival from radiation-associated angiosarcoma of the breast. Br J Surg 105(4): 401-409
4. Pencavel T, Allan CP, Thomas JM, Hayes AJ (2011) Treatment for breast sarcoma: a large, single-centre series. Eur J Surg Oncol 37(8): 703-708

5. Scottish Sarcoma Network Guidelines (2004): https://www.ssn.scot. nhs.uk/guidelines/. Accessed 05 Feb 2019

6. Kadouri L, Sagi M, Goldberg Y (2013) Genetic predisposition to radiation induced sarcoma: possible role for BRCA and p53 mutations. Breast Cancer Res Treat 140(1):207-211

7. Barbosa OV, Reiriz AB, Boff RA, Oliveira WP, Rossi L (2015) Angiosarcoma in previously irradiated breast in patient with LiFraumeni syndrome. A case report. Sao Paulo Med J 133(2):151153

8. West JG, Weizel JN, Tao ML, Carpenter M, West JE, Fanning C (2008) BRCA mutations and the risk of angiosarcoma after breast cancer treatment. Clin Breast Cancer 8(6):533-537

Publisher's note Springer Nature remains neutral with regard to jurisdictional claims in published maps and institutional affiliations. 